University of Nebraska - Lincoln

DigitalCommons@University of Nebraska - Lincoln

SPECTRA OF TYPE II CEPHEID CANDIDATES AND RELATED STARS

\author{
Edward G. Schmidt \\ University of Nebraska at Lincoln, eschmidt1@unl.edu \\ Danielle Rogalla \\ University of Nebraska - Lincoln, drogall1@bigred.unl.edu \\ Lauren Thacker-Lynn \\ University of Nebraska - Lincoln, Ithacke1@bigred.unl.edu
}

Follow this and additional works at: https://digitalcommons.unl.edu/physicsschmidt

Part of the Physics Commons

Schmidt, Edward G.; Rogalla, Danielle; and Thacker-Lynn, Lauren, "SPECTRA OF TYPE II CEPHEID CANDIDATES AND RELATED STARS" (2011). Edward Schmidt Publications. 50.

https://digitalcommons.unl.edu/physicsschmidt/50

This Article is brought to you for free and open access by the Research Papers in Physics and Astronomy at DigitalCommons@University of Nebraska - Lincoln. It has been accepted for inclusion in Edward Schmidt Publications by an authorized administrator of DigitalCommons@University of Nebraska - Lincoln. 


\title{
SPECTRA OF TYPE II CEPHEID CANDIDATES AND RELATED STARS
}

\author{
E. G. Schmidt ${ }^{1}$, Danielle Rogalla ${ }^{1}$, AND Lauren ThaCKer-LynN ${ }^{1}$ \\ Department of Physics and Astronomy, University of Nebraska, Lincoln, NE 68588-0299, USA; \\ eschmidt1@unl.edu,drogall1@bigred.unl.edu, lologur1329@aol.com \\ Received 2010 October 26; accepted 2010 November 6; published 2011 January 13
}

\begin{abstract}
We present low-resolution spectra for variable stars in the Cepheid period range from the ROTSE-I Demonstration Project and the All Sky Automated Survey, some of which were previously identified as type II Cepheid candidates. We have derived effective temperatures, gravities, and metallicities from the spectra. Based on this, three types of variables were identified: Cepheid strip stars, cool stars that lie along the red subgiant and giant branch, and cool main-sequence stars. Many fewer type II Cepheids were found than expected and most have amplitudes less than $0.4 \mathrm{mag}$. The cool variables include many likely binaries as well as intrinsic variables. Variation among the main-sequence stars is likely to be mostly due to binarity or stellar activity.
\end{abstract}

Key words: stars: Population II - stars: variables: Cepheids

Online-only material: machine-readable and VO tables

\section{INTRODUCTION}

Type II Cepheid variable stars are potentially useful to a variety of areas of astrophysics including studies of the structure of the Galactic thick disk and halo, stellar pulsation, and the late stages of stellar evolution. However, their application has been limited by the relatively small number of these stars known in the Milky Way, about 200. On the other hand, Wallerstein (2002) argued that there should be about 6000 undiscovered type II Cepheids on the basis of the number known in globular clusters. We have undertaken a search for these objects based on the results of recent large area surveys.

Schmidt et al. (2007, 2009, Papers I and II, respectively) presented photometry of 487 Cepheid candidates selected from the ROTSE-I Demonstration Project (Akerlof et al. 2000) and the northern portion of the All Sky Automated Survey (ASAS; Pojmanski et al. 2005). The new photometry, in combination with the survey data allowed us to identify 19 new probable type II Cepheids. Considering the limiting magnitudes and sky coverage of the two surveys, we should revise Wallerstein's estimate to about 560 type II Cepheids for our sample; the number found falls far short of this expectation.

It is likely that other type II Cepheids are to be found in this sample, particularly if small-amplitude type II Cepheids exist. Nonetheless, it is clear that a large majority of the stars are of other types (see, for example, Figures 1 and 4 of Paper II). In this paper, stellar parameters derived from spectra are used to clarify the nature of these stars and confirm the identification of some as type II Cepheids.

\section{THE OBSERVATIONS}

\subsection{The Spectra}

We obtained spectra for 288 stars from Papers I and II as well as 35 known Cepheids and 30 HD stars. The latter, referred to as "standard stars" below, were included to permit the matching of our indices to previous data as discussed in Section 2.3. For

\footnotetext{
1 Visiting Astronomer, Kitt Peak National Observatory, National Optical Astronomy Observatory, which is operated by the Association of Universities for Research in Astronomy, Inc., under cooperative agreement with the National Science Foundation.
}

most of the stars we obtained at least two spectra on different nights.

The spectra were obtained at Kitt Peak National Observatory during 14 nights in 2006 June, 2009 January, and 2009 April using the GoldCam Spectrograph on the $2.1 \mathrm{~m}$ telescope (Massey et al. 2000). ${ }^{2}$ Grating 240 was used in first order to produce a dispersion of $1.5 \AA$ pixel $^{-1}$ and a resulting resolution of $\sim 3.7 \AA$. The useful spectral coverage extended from approximately $4000 \AA$ to $6800 \AA$ A. The IRAF package ${ }^{3}$ was used to reduce the spectra following standard procedures.

We tried to achieve a signal-to-noise ratio of $\mathrm{S} / \mathrm{N} \sim 100$ per pixel at $5300 \AA$ for stars brighter than $V=13.7$. For fainter stars, we adopted a constant exposure time of 10 minutes to avoid compromising our goal of obtaining as complete a sample of the stars from Papers I and II as possible. In practice, there is a range in $\mathrm{S} / \mathrm{N}$ even among the brighter stars due to variations in transparency, seeing, air mass, etc.

Table 1 contains the log of the spectral observations. The first and second columns identify the stars and list their periods, while the third column gives the $V$ amplitudes. The identifiers come from Papers I and II for stars discussed there and from the GCVS for known variables. The periods and amplitudes were taken from Papers I and II for stars discussed there and from Schmidt et al. (2004a, 2005a, 2005b) for the known variables. Column 4 lists the heliocentric Julian Date at mid-exposure for each spectrum. The remaining columns are discussed below. The stars are listed in the four groups defined in Paper II followed by stars not associated with any group and known Cepheids.

\subsection{Calculation of the Lick/IDS Indices}

To derive the atmospheric parameters from the spectra, we calculated Lick/IDS indices, as defined by Worthey et al. (1994) and Worthey \& Ottaviani (1997), from the spectra.

Some of the stars in our sample are likely to have large radial velocities that could affect the calculated indices. To deal with this, each spectrum was reduced to the rest frame of the

\footnotetext{
2 Available at http://www.noao.edu/kpno/manuals/12mspect/ spectroscopy.html.

3 IRAF is distributed by the National Optical Astronomy Observatory, which is operated by the Association for Research in Astronomy, Inc., under cooperative agreement with the National Science Foundation.
} 
Table 1

Log of Observations

\begin{tabular}{|c|c|c|c|c|c|c|c|c|}
\hline Star & $\begin{array}{c}\text { Period } \\
\text { (2) }\end{array}$ & $\Delta V$ & $\begin{array}{c}\text { Mid-Exposure } \\
\text { HJD - } 2,450,000 \\
(4) \\
\end{array}$ & $\begin{array}{l}T_{\text {eff }} \\
(5) \\
\end{array}$ & $\log g$ & {$[\mathrm{Fe} / \mathrm{H}]$} & $\begin{array}{c}E_{V-R} \\
(8)\end{array}$ & Notes \\
\hline \multicolumn{9}{|c|}{ Group 1} \\
\hline A129 & 0.95246 & 0.10 & 4840.00 & 4616 & 3.28 & 0.18 & 0.02 & \\
\hline A129 & 0.95246 & 0.10 & 4930.78 & 4604 & 3.20 & 0.18 & 0.02 & \\
\hline A196 & 1.1075 & 0.05 & 4931.97 & 5197 & 4.50 & -0.18 & 0.12 & \\
\hline A019 & 1.156 & 0.07 & 4838.70 & 4868 & 4.60 & -0.10 & 0.01 & \\
\hline A019 & 1.156 & 0.07 & 4840.64 & 4853 & 4.60 & -0.10 & 0.00 & \\
\hline A019 & 1.156 & 0.07 & 4841.69 & 4892 & 4.58 & -0.03 & 0.01 & \\
\hline A224 & 1.2775 & 0.07 & 4934.98 & 4706 & 4.57 & -0.02 & 0.03 & \\
\hline R012 & 1.38535 & 0.15 & 3903.77 & 5253 & 4.45 & 0.04 & 0.08 & \\
\hline R013 & 1.4334 & 0.16 & 3902.84 & 5186 & 4.19 & -0.32 & 0.03 & \\
\hline
\end{tabular}

(This table is available in its entirety in machine-readable and Virtual Observatory (VO) forms in the online journal. A portion is shown here for guidance regarding its form and content.)

spectrograph using an HeNeAr arc spectrum taken immediately before or after the stellar spectrum. We then measured the shifts of a selection of spectral lines relative to that frame. The relative velocities were used to correct the wavelength scales of the spectra to the rest frame of the stellar atmosphere prior to calculating the Lick/IDS indices.

The number of lines that can be measured on various spectra depends on the temperature and metallicity but averaged about 10. The average standard error of the relative radial velocities was $7 \mathrm{~km} \mathrm{~s}^{-1}$, corresponding to between $0.09 \AA$ and $0.16 \AA$. This is sufficiently small to ensure that the Lick/IDS indices are unaffected.

Since the present spectra are of higher resolution than those used to define the Lick/IDS system, we convolved them with a Gaussian of variable width following the recommendation of Worthey \& Ottaviani (1997). The indices were then calculated using the definitions of Worthey et al. (1994) and Worthey \& Ottaviani (1997). We added an additional index to measure the strength of $\mathrm{H} \alpha$. For that index, the central band was taken to be $\lambda$ 6553-6573 with pseudo-continuum bands of $\lambda$ 6506-6540 and $\lambda$ 6586-6620.

The indices are listed in Table 2 where the first column lists the star name and the second the Julian Date of the spectrum. The 26 indices are contained in the remaining columns. For completeness, the indices of the standard stars are also included.

We estimated a characteristic uncertainty for each index from the scatter for stars with multiple spectra. The $\mathrm{S} / \mathrm{N}$ of the spectra of the standard stars is much larger than for the program stars and they were excluded from this calculation. The known Cepheids were also excluded because their large amplitudes will artificially increase the scatter. The median standard deviations of the indices from individual spectra, $\sigma_{i}$, are listed in Columns 2,5 , and 8 of Table 3 . The use of the median mitigates the effects of variability to some degree but the errors may still be overestimated. We have also listed the relative errors of each index, $\sigma_{i} / r_{i}$, in Columns 3,6, and 9, where $r_{i}$ is the range of index $i$ over all the MILES reference stars described below. The relative errors fall in the range from less than one percent to three percent.

\subsection{Comparison to Previous Measurements}

As described below, we interpreted our data by reference to indices calculated from the MILES survey spectra (Sánchez-
Blázquez et al. 2006; Cenarro et al. 2007). We derived linear transformations between our indices and the MILES system using 35 spectra of 16 standard stars in common between the two data sets. The coefficients of the transformations are displayed on the left side of Table 4. These can be used to transform the data in Table 2 to the MILES system. All except two of the standard stars are too hot to exhibit $\mathrm{TiO}$ bands. We therefore assumed transformation slopes of unity for $\mathrm{TiO}_{1}$ and $\mathrm{TiO}_{2}$. The listed values of $\sigma_{i}$ represent the scatter of individual points about the transformations. Since they include errors in both our measured indices and the values from the MILES list, they are generally somewhat larger than the internal errors listed in Table 3.

No standard stars were obtained during the 2006 observing run. However, a comparison of the standard star indices for stars in common between the two runs in 2009 showed a negligible difference. Hence, we have assumed the same transformations for the 2006 spectra. As an additional test, we analyzed our program stars as described in Section 3.2, using both the raw indices and transformed indices. The mean differences were $2 \mathrm{~K} \pm 232 \mathrm{~K}$ (or $16 \mathrm{~K} \pm 102 \mathrm{~K}$ deleting 7 discrepant points out of nearly 700), $0.12 \pm 0.29$, and $0.04 \pm 0.13$ for $T_{\text {eff }}, \log g$, and $[\mathrm{Fe} / \mathrm{H}]$, respectively, where the uncertainties are the standard deviation for a single spectrum. Thus, the systematic effects of the transformations and any uncertainties in them are negligible.

\section{ANALYSIS OF THE SPECTRA}

\subsection{Hydrogen Line Emission}

A cursory examination of the hydrogen indices or the spectra themselves reveals that emission in the hydrogen lines, particularly in $\mathrm{H} \alpha$, is relatively common. This is illustrated in Figure 1 which shows some examples of spectra around $\mathrm{H} \beta$ and $\mathrm{H} \alpha$. The plots are arranged from top to bottom in order of the $\mathrm{H} \alpha$ index and cover the range exhibited by the program stars.

The line indices correlate well with the appearance of the lines; in both $\mathrm{H} \alpha$ and $\mathrm{H} \beta$, an index near 0.0 corresponds to a spectrum in which the absorption is just filled by emission, leaving little evidence of the line.

We were able to find no significant evidence of emission in the higher Balmer lines and can safely assume they are affected by emission to a negligible extent.

\subsection{The Derivation of Atmospheric Parameters}

Worthey \& Jowett (2003) devised a method to use the fitting functions of Worthey et al. (1994) and Worthey \& Ottaviani (1997) to derive atmospheric parameters from Lick/IDS indices. Robinson et al. (2006) explored the reliability of this method for dwarfs in the temperature range of interest here and found significant scatter and systematic errors. They speculated that at least part of the problem may be that the fitting functions were not well behaved in some parameter ranges. To avoid such problems, we have adopted an approach that compares the Lick/IDS indices from our spectra directly to those from a set of reference stars with known atmospheric parameters derived from high-resolution spectra.

Worthey et al. (1994) and Worthey \& Ottaviani (1997) have provided indices for a large number of stars with known atmospheric parameters. ${ }^{4}$ Although this data set defines the Lick/IDS system, the MILES survey was selected instead as

\footnotetext{
4 Available at http://astro.wsu.edu/worthey/html/system.html. Hereafter referred to as the Worthey Data Set.
} 
Table 2

LICK/IDS Indices

\begin{tabular}{lccccccccc}
\hline \hline Star & $\begin{array}{c}\text { Mid-Exposure } \\
\text { HJD - 2,450,000 }\end{array}$ & $\mathrm{CN}_{1}$ & $\mathrm{CN}_{2}$ & $\mathrm{Ca} 4227$ & $\mathrm{G} 4300$ & $\mathrm{Fe} 4383$ & $\mathrm{Ca} 4455$ & $\mathrm{Fe} 4531$ & $\mathrm{Fe} 4668$ \\
$(1)$ & $(2)$ & $(3)$ & $(4)$ & $(5)$ & $(6)$ & $(7)$ & $(8)$ & $(9)$ & $(10)$ \\
\hline $\mathrm{A} 129$ & 4840.00 & 0.206 & 0.230 & 1.645 & 6.527 & 6.851 & 1.169 & 4.054 & 9.591 \\
$\mathrm{~A} 129$ & 4930.78 & 0.228 & 0.256 & 1.475 & 6.190 & 6.751 & 1.510 & 3.892 & 9.300 \\
$\mathrm{~A} 196$ & 4931.97 & -0.016 & 0.002 & 1.207 & 6.311 & 4.401 & 0.387 & 3.323 & 3.081 \\
$\mathrm{~A} 019$ & 4838.70 & 0.006 & 0.017 & 1.755 & 6.049 & 6.046 & 0.652 & 3.981 & 4.528 \\
$\mathrm{~A} 019$ & 4840.64 & 0.003 & 0.020 & 2.024 & 5.793 & 6.417 & 1.033 & 4.061 & 4.271 \\
$\mathrm{~A} 019$ & 4841.69 & -0.014 & 0.005 & 2.282 & 6.249 & 5.835 & 0.812 & 4.397 & 4.257 \\
$\mathrm{~A} 224$ & 4934.98 & -0.004 & 0.017 & 2.633 & 5.810 & 6.543 & 0.857 & 4.247 & 3.641 \\
$\mathrm{R} 012$ & 3903.77 & -0.010 & -0.003 & 1.384 & 5.423 & 5.440 & 0.950 & 3.734 & 5.551 \\
$\mathrm{R} 013$ & 3902.84 & -0.023 & -0.013 & 1.247 & 5.488 & 4.509 & 0.854 & 3.460 & 3.381 \\
\hline
\end{tabular}

Notes. The electronic version contains an additional 18 columns to accommodate all of the LICK/ IDS indices.

(This table is available in its entirety in machine-readable and Virtual Observatory (VO) forms in the online journal. A portion is shown here for guidance regarding its form and content.)

Table 3

Typical Internal Errors in the LICK/IDS Indices and Atmospheric Parameters

\begin{tabular}{lcccccccc}
\hline \hline Index & $\sigma_{i}$ & $\sigma_{i} / r_{i}(\%)$ & $\operatorname{Index}$ & $\sigma_{i}$ & $\sigma_{i} / r_{i}(\%)$ & Index & $\sigma_{i}$ & $\sigma_{i} / r_{i}(\%)$ \\
$(1)$ & $(2)$ & $(3)$ & $(4)$ & $(5)$ & $(6)$ & $(7)$ & $(8)$ & $(9)$ \\
\hline $\mathrm{CN}_{1}$ & 0.010 & 0.9 & $\mathrm{Fe} 5015$ & 0.219 & 0.8 & $\mathrm{Na} \mathrm{D}$ & 0.167 & 1.4 \\
$\mathrm{CN}_{2}$ & 0.010 & 0.8 & $\mathrm{Mg}_{1}$ & 0.004 & 0.8 & $\mathrm{TiO}_{1}$ & 0.005 & 0.9 \\
$\mathrm{Ca}_{2227}$ & 0.114 & 1.4 & $\mathrm{Mg}_{2}$ & 0.006 & 1.2 & $\mathrm{TiO}_{2}$ & 0.004 & 0.4 \\
$\mathrm{G} 4300$ & 0.205 & 1.5 & $\mathrm{Mg} b$ & 0.128 & 0.8 & $\mathrm{H} \delta_{A}$ & 0.492 & 1.8 \\
$\mathrm{Fe} 4383$ & 0.248 & 1.2 & $\mathrm{Fe} 5269$ & 0.114 & 2.0 & $\mathrm{H} \gamma_{A}$ & 0.361 & 1.2 \\
$\mathrm{Ca} 4455$ & 0.129 & 2.7 & $\mathrm{Fe} 5335$ & 0.112 & 2.4 & $\mathrm{H} \delta_{F}$ & 0.276 & 1.3 \\
$\mathrm{Fe} 4531$ & 0.195 & 2.7 & $\mathrm{Fe} 5406$ & 0.086 & 2.4 & $\mathrm{H} \gamma_{F}$ & 0.182 & 0.7 \\
$\mathrm{Fe} 4668$ & 0.323 & 1.1 & $\mathrm{Fe} 5709$ & 0.096 & 1.9 & $\mathrm{H} \alpha$ & 0.167 & $\cdots$ \\
$\mathrm{H} \beta$ & 0.159 & 0.8 & $\mathrm{Fe} 5782$ & 0.066 & 3.0 & & & \\
$T_{\text {eff }}$ & $61 \mathrm{~K}$ & & $\log g$ & 0.16 & & {$[\mathrm{Fe} / \mathrm{H}]$} & 0.06 & \\
& & & $E_{(V-R)}$ & 0.02 & & & & \\
\hline
\end{tabular}

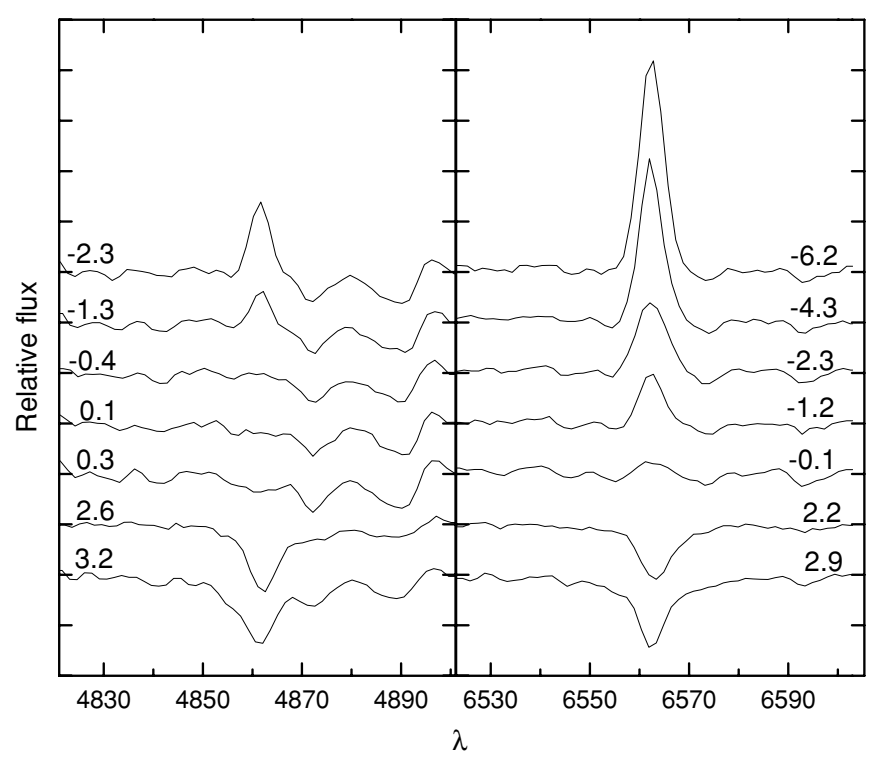

Figure 1. $\mathrm{H} \alpha$ and $\mathrm{H} \beta$ regions of selected spectra. Each spectrum was normalized to a continuum level of 1.0 and each is offset vertically from its neighbors by 0.25 for visibility. From top to bottom, the spectra are for the stars and HJD 2,453,900 as follows: R030, 3.96; R030, 1.88; R037, 4.73; R043, 3.81; R101, $3.90 ; \mathrm{R} 039,5.75 ; \mathrm{R} 168,5.70$. The $\mathrm{H} \alpha$ index is at the right and the $\mathrm{H} \beta$ index at the left above each spectrum.

the reference set for two reasons: the scatter of the standard stars about the transformations was significantly smaller when our data were compared the indices from MILES than when compared to the Worthey indices, and the MILES survey provided many more reference stars, 843 as compared to 245 .

Considering the parameter space defined by $N$ indices, we seek the MILES stars that are nearest to each program star in that space. The distance of a program star, $p$, from a MILES reference star, $r$, is defined by

$$
r_{p, r}^{2}=\sum_{i=1}^{N} \frac{\left(I_{p, i}-I_{r, i}\right)^{2}}{\delta_{i}^{2}}
$$

where $I_{p, i}$ and $I_{r, i}$ are the measured values for index $i$ for each star and

$$
\delta_{i}^{2}=\frac{\sum_{r}\left(I_{r, i}-\bar{I}_{i}\right)^{2}}{N}
$$

is the dispersion of index $i$ among the MILES stars, a measure of the range of the index. To reduce the effect of errors in the assumed atmospheric parameters of the MILES stars, we have averaged the values for the three reference stars nearest each program star and assigned them to that star.

Jones et al. (1995), among others, have pointed out a degeneracy among $T_{\text {eff }}, \log g$, and $[\mathrm{Fe} / \mathrm{H}]$ in spectral indices. This occurs because both the temperature and the gravity affect the ionization; an increase in one can be compensated by a decrease in the other to produce the same value for an index. Similarly, changes in temperature and abundance both affect an index and can compensate each other. In the present case, the degeneracy 
would be manifest by stars in the reference set that lie close together in index space but have significantly different atmospheric parameters.

For cluster stars, the degeneracy is often addressed by using a color index to provide the temperature and then deriving log $g$ and $[\mathrm{Fe} / \mathrm{H}]$ from the spectral indices (see, for example, Friel 1987). In the present case, this is problematic because we are studying field stars for which the colors are reddened by an unknown amount.

Nonetheless, the observed colors can be used to remove the effect of the degeneracy in some cases. We define the color excess implied by reference star $r$ as $E_{V-R, r}=(V-R)_{p}-$ $(V-R)_{r}$, where $(V-R)_{p}$ is the observed color of the program star and $(V-R)_{r}$ is the color implied by the atmospheric parameters of the reference star. A negative value of $E_{V-R, r}$ indicates that the reference star is inappropriate for the program star. In practice, this was relaxed to $E_{V-R, r}>-0.05$ to allow for some uncertainty in the observed and calculated colors. Any reference star that violated this condition was not used for program star $p$. $(V-R)_{p}$ at the pulsational phases of the spectra came from the color curves of Schmidt et al. (2004a, 2005a, 2005b) and Papers I and II. $(V-R)_{r}$ was calculated using the tables of VandenBerg \& Clem (2003).

The following 14 indices were used in the derivation of the atmospheric parameters from our spectra: $\mathrm{CN}_{2}, \mathrm{G} 4300, \mathrm{Fe} 4383$, Fe4531, Fe4668, Fe5015, $\mathrm{Mg}_{b}$, Fe5270, Fe5335, Fe5406, Na D, $\mathrm{TiO}_{1}, \mathrm{H} \delta_{F}$, and $\mathrm{H} \gamma_{F} . \mathrm{CN}_{1}, \mathrm{Mg}_{1}, \mathrm{Mg}_{2}, \mathrm{TiO}_{2}, \mathrm{H} \delta_{A}$, and $\mathrm{H} \gamma_{A}$ were omitted because they measure the same spectral features as other indices and thus add no information. Ca4227, Ca4455, Fe5709, and Fe5782 were left out because they did not transform to the MILES system as well as the other indices (see Table 4). As discussed above, a significant number of program stars exhibit emission in $\mathrm{H} \alpha$ and sometimes in $\mathrm{H} \beta$. Accordingly, the $\mathrm{H} \beta$ index was not used.

Table 1 lists the adopted atmospheric parameters in Columns 5,6 , and 7. We have also calculated color excesses for all of the stars as described above for $E_{V-R, r}$ and they are listed in Column 8.

\subsection{Validation of the Analysis}

We have performed several tests of the results to understand the limitations and uncertainties of our method.

\subsubsection{The Internal Consistency}

The indices are sensitive to the atmospheric parameters in varying degrees in various regions of parameter space. Additionally, as noted above, the uncertainties of the indices will vary from spectrum to spectrum and will become larger at magnitudes below $V=13.7$. We have again calculated typical errors using stars observed more than once. The median errors are listed in the lower part of Table 3. The internal consistency of our results is quite acceptable for our purposes.

\subsubsection{The Worthey Data Set}

The Worthey Data Set lists Lick/IDS indices for 245 stars for which all three atmospheric parameters have been determined from high-resolution spectra. To test the above method, we transformed these indices to the MILES system using the 135 stars in common. Information on these transformations are displayed on the right side of Table $4 .^{5} V-R$ colors for

\footnotetext{
5 A comparison of Column 5 to Column 9 illustrates the poorer agreement between the Worthey and the MILES indices as compared with that between the present indices and the MILES indices as noted in Section 3.2.
}

Table 4

Transformations Between Systems of Spectral Indices

\begin{tabular}{|c|c|c|c|c|c|c|c|c|}
\hline \multirow[t]{2}{*}{ Index } & \multicolumn{3}{|c|}{$\begin{array}{c}\text { Present Indices to } \\
\text { MILES }\end{array}$} & \multicolumn{5}{|c|}{ Worthey to MILES } \\
\hline & Slope & Intercept & $\sigma_{i}$ & $\begin{array}{c}\sigma_{i} / r_{i} \\
(\%)\end{array}$ & Slope & Intercept & $\sigma_{i}$ & $\begin{array}{c}\sigma_{i} / r_{i} \\
(\%)\end{array}$ \\
\hline (1) & (2) & (3) & (4) & (5) & (6) & (7) & (8) & $(9)$ \\
\hline $\mathrm{CN}_{1}$ & 0.980 & -0.004 & 0.014 & 1.6 & 0.948 & 0.008 & 0.027 & 3.2 \\
\hline $\mathrm{CN}_{2}$ & 0.973 & -0.019 & 0.016 & 1.9 & 0.960 & 0.069 & 0.029 & 3.5 \\
\hline $\mathrm{Ca} 4227$ & 1.296 & -0.060 & 0.143 & 1.8 & 0.941 & -0.017 & 0.317 & 3.9 \\
\hline G4300 & 0.895 & 0.399 & 0.203 & 1.5 & 0.880 & 0.459 & 0.475 & 3.6 \\
\hline $\mathrm{Fe} 4383$ & 1.090 & 0.015 & 0.234 & 1.2 & 1.001 & -0.434 & 0.755 & 3.8 \\
\hline $\mathrm{Ca} 4455$ & 1.440 & 0.019 & 0.178 & 3.8 & 0.984 & -0.400 & 0.424 & 8.9 \\
\hline Fe4531 & 1.055 & -0.050 & 0.170 & 2.4 & 0.922 & 0.130 & 0.447 & 6.2 \\
\hline Fe4668 & 0.966 & 0.644 & 0.383 & 1.3 & 0.926 & 0.408 & 0.681 & 2.3 \\
\hline $\mathrm{H} \beta$ & 1.033 & 0.025 & 0.098 & 0.5 & 1.025 & 0.059 & 0.292 & 1.6 \\
\hline Fe5015 & 0.978 & 0.038 & 0.200 & 0.7 & 0.937 & -0.141 & 0.605 & 2.2 \\
\hline $\mathrm{Mg}_{1}$ & 0.970 & 0.018 & 0.009 & 1.9 & 0.939 & -0.004 & 0.010 & 2.1 \\
\hline $\mathrm{Mg}_{2}$ & 0.968 & 0.016 & 0.008 & 1.6 & 0.927 & -0.005 & 0.011 & 2.2 \\
\hline $\operatorname{Mg} b$ & 0.997 & -0.018 & 0.191 & 1.2 & 0.868 & 0.032 & 0.359 & 2.3 \\
\hline Fe5270 & 0.986 & 0.036 & 0.135 & 2.3 & 0.935 & 0.053 & 0.282 & 4.8 \\
\hline Fe5335 & 0.975 & 0.137 & 0.092 & 2.0 & 0.973 & 0.016 & 0.316 & 6.8 \\
\hline Fe5406 & 1.015 & 0.053 & 0.047 & 1.3 & 0.937 & 0.143 & 0.206 & 5.7 \\
\hline Fe5709 & 1.274 & -0.031 & 0.080 & 1.6 & 1.043 & -0.065 & 0.216 & 4.2 \\
\hline Fe5782 & 1.253 & -0.051 & 0.071 & 3.2 & 1.047 & -0.059 & 0.159 & 7.2 \\
\hline $\mathrm{NaD}$ & 1.032 & -0.304 & 0.173 & 1.5 & 1.032 & -0.291 & 0.333 & 2.8 \\
\hline $\mathrm{TiO}_{1}$ & $1.000^{\mathrm{a}}$ & $0.000^{\mathrm{a}}$ & 0.007 & 1.3 & 0.917 & -0.005 & 0.009 & 1.7 \\
\hline $\mathrm{TiO}_{2}$ & $1.000^{\mathrm{a}}$ & $0.000^{\mathrm{a}}$ & 0.007 & 0.8 & 0.918 & 0.000 & 0.013 & 1.4 \\
\hline $\mathrm{H} \delta_{A}$ & 0.964 & -0.355 & 0.267 & 1.0 & 0.948 & 0.089 & 0.769 & 2.9 \\
\hline $\mathrm{H} \gamma_{A}$ & 0.987 & -0.275 & 0.298 & 1.0 & 0.989 & -0.153 & 0.698 & 2.3 \\
\hline $\mathrm{H} \delta_{F}$ & 0.995 & -0.072 & 0.128 & 0.6 & 0.998 & 0.026 & 0.428 & 2.0 \\
\hline $\mathrm{H} \gamma_{F}$ & 1.024 & -0.071 & 0.114 & 0.4 & 1.004 & -0.089 & 0.432 & 1.6 \\
\hline
\end{tabular}

Note. ${ }^{\text {a }}$ Assumed.

many of the Worthey stars are not available. Accordingly, the atmospheric parameters were derived as described above except for the use of color indices to eliminate the effects of degeneracy.

In Figure 2, the differences between each of the three atmospheric parameters derived from the Lick/IDS indices and those from the high-resolution spectral studies are plotted (in the sense of Lick/IDS-high resolution) against $T_{\text {eff }}$ from the high-resolution studies. Eliminating three points that fall outside the range of Figure 2(a) due to anomalous temperatures, the standard deviations of $\Delta T_{\text {eff }}, \Delta \log g$, and $\Delta[\mathrm{Fe} / \mathrm{H}]$ are $226 \mathrm{~K}, 0.55$, and 0.37 , respectively. We will take these to be conservative estimates of the uncertainties in our derived parameters.

\subsubsection{Atmospheric Parameters of the Known Cepheids and Standard Stars}

In Figure 3, we plot the atmospheric parameters from Table 1 for the known Cepheids and the standard stars against values from high-resolution spectral studies. The latter were taken from Kovtyukh et al. (2005), Maas et al. (2007), and Wallerstein et al. (2008) for the Cepheids and from the MILES and Worthey tables for the standard stars. In panel (c), we also include photometric abundances from Harris (1981a, 1981b) and Diethelm (1990) for Cepheids.

Omitting an outlier in temperature (from UY Eri) that falls outside the area of the plot, our parameters and those from high-resolution spectra differ systematically by $480 \mathrm{~K} \pm 403 \mathrm{~K}$ (standard deviation of a single point), $1.18 \pm 0.89$, and $0.35 \pm$ 0.31 for $T_{\text {eff }}, \log g$, and [Fe/H], respectively, for the Cepheids. The metallicities of Harris (1981a, 1981b) agree well with the 


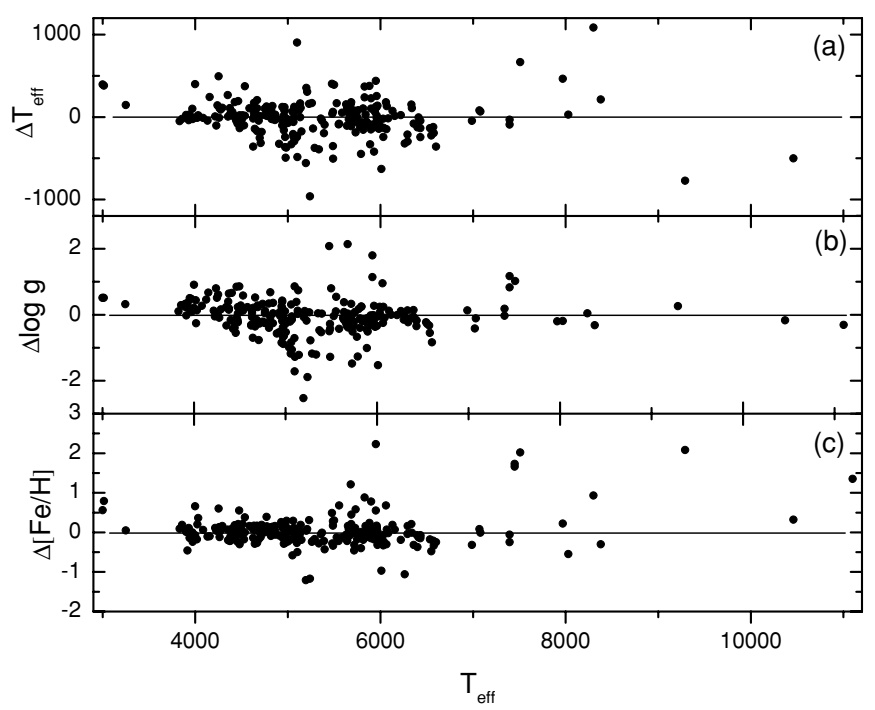

Figure 2. Difference between the atmospheric parameters derived from the Lick/IDS indices and those from high-resolution spectra plotted against $T_{\text {eff }}$ for the stars from Worthey's Web site.

high-resolution results while those of Diethelm (1990), although few in number, agree well with our values. On the other hand, our atmospheric parameters for the standard stars and those from high dispersion studies agree considerably better with mean differences of $88 \mathrm{~K} \pm 305 \mathrm{~K}, 0.01 \pm 0.27$, and $0.13 \pm 0.35$, respectively.

The zero-point shifts between our results and previous ones for the Cepheids are surprising in view of the excellent agreement for the standard stars and between the values we derived for the Worthey stars and those from high-resolution studies. A detailed discussion of these discrepancies is outside the scope of this paper and will be addressed later. However, in interpreting our results below, they must be kept in mind.

\subsubsection{Color Excesses of the Known Cepheids}

Color excesses determined from high-resolution spectra were published by Kovtyukh et al. (2008), while Fernie's Database of Galactic Classical Cepheids ${ }^{6}$ lists mean color excesses

6 http://www.astro.utoronto.ca/DDO/research/cepheids/

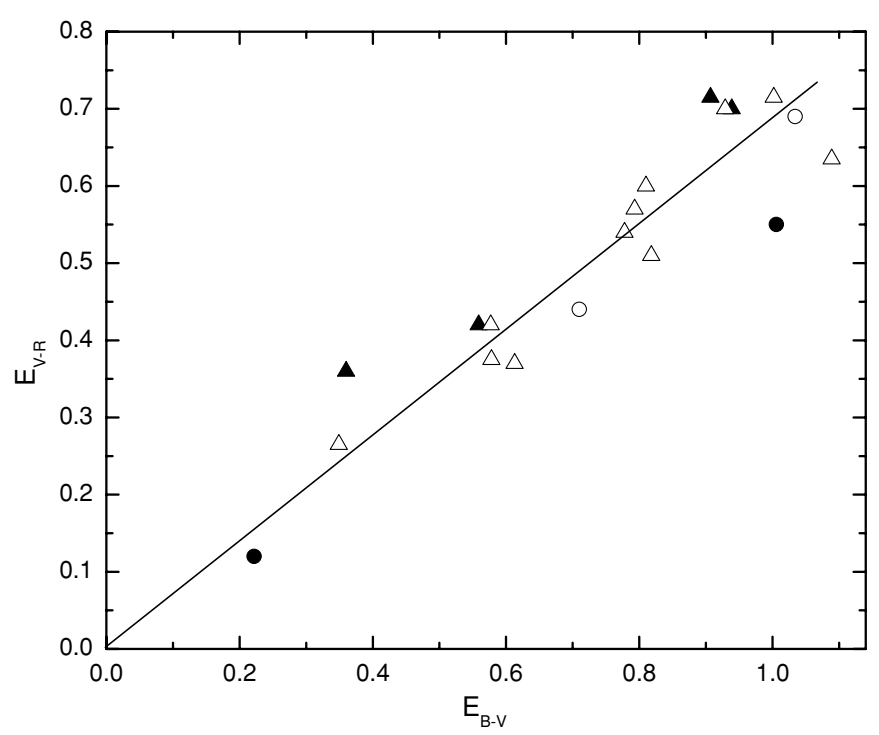

Figure 4. Color excesses from this study plotted against values from Kovtyukh et al. (2008), filled symbols, and from the Database of Galactic Classical Cepheids, open symbols. Triangles denote classical Cepheids and circles denote type II Cepheids.

from numerous sources for a large number of Cepheids. In Figure 4 , we have plotted our color excesses, $E_{V-R}$, against $E_{B-V}$ from those sources for the stars in common. The plotted line has a slope of 0.68 and the rms scatter of the points around it is $0.06 \mathrm{mag}$. Both these values are satisfactory and support the consistency of our analysis with previous work. This also shows that the color excesses are adequate for correcting photometry for interstellar absorption.

\section{DISCUSSION}

In Figure 5(a), we have plotted the gravities versus the temperatures for all the spectra from Table 1 . The axes are inverted so as to resemble an Hertzsprung-Russell diagram. Stars from the different categories in Table 1 are represented by different symbols as indicated in the figure caption. The measured temperatures have been corrected to the mean temperature, $\left\langle T_{\mathrm{eff}}\right\rangle$, using the measured color at the phase of the spectrum, $(V-R)_{\phi}$, and the mean color over the cycle, $\langle V-R\rangle$, along with the
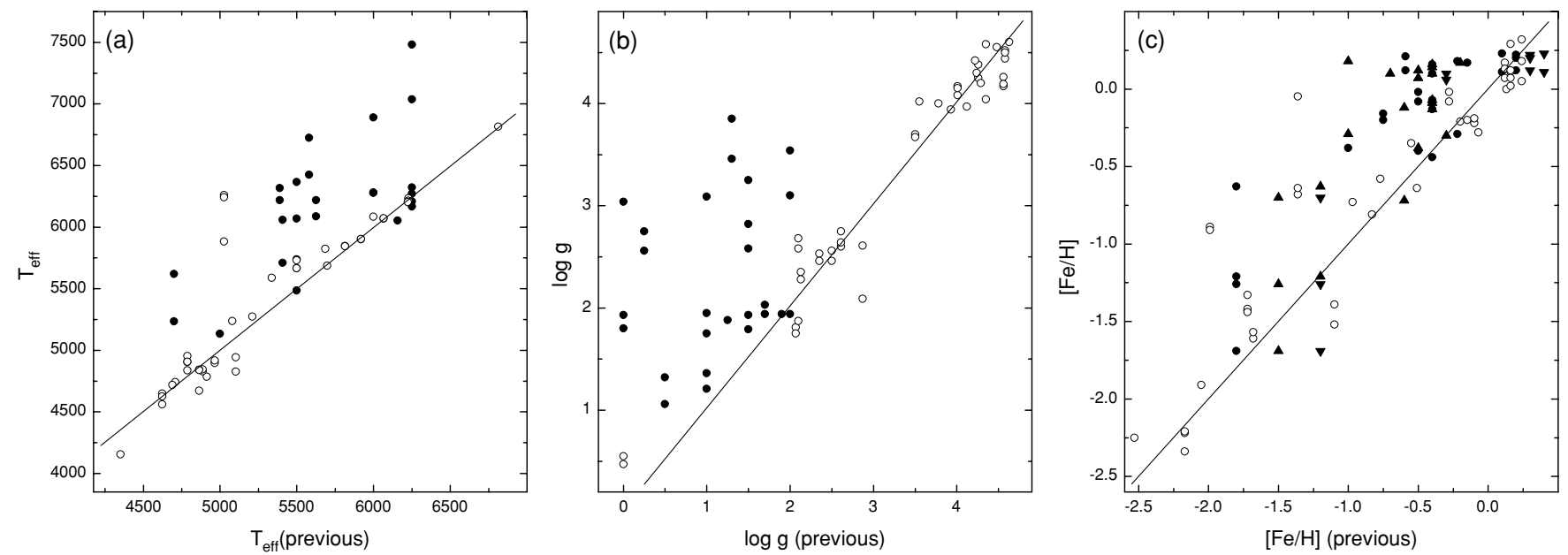

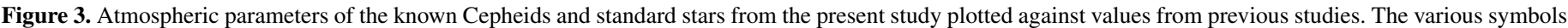

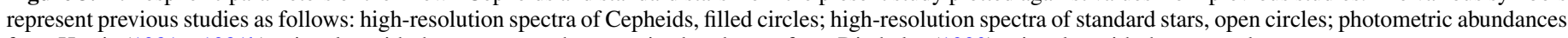
from Harris (1981a, 1981b), triangles with the vertex up; photometric abundances from Diethelm (1990), triangles with the vertex down. 

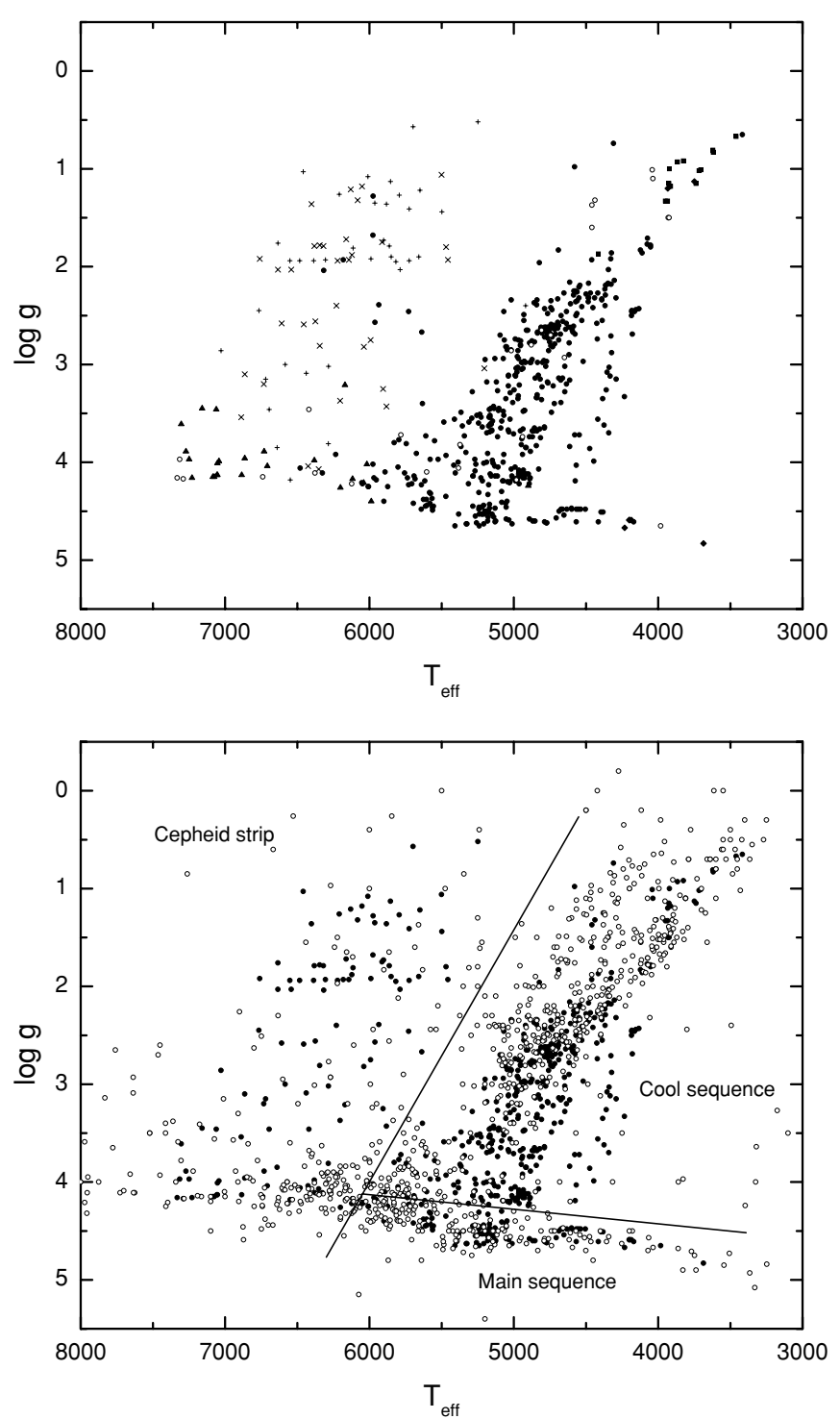

Figure 5. $\log g$ plotted against $T_{\text {eff. In panel (a) the symbols indicate stars }}$ from the various groups in Table 1 as follows: stars from group 1, solid circles; stars from group 2, triangles; stars from group 3, squares; stars from group 4, diamonds; program stars not in any group, open circles; known classical Cepheids, plus signs; known type II Cepheids, Xs. In panel (b) filled circles indicate stars from Table 1, while open circles denote reference stars from the MILES survey. The solid lines divide the sample into three types of stars as discussed in the text.

color-temperature relations of VandenBerg \& Clem (2003). The photometry was taken from Schmidt et al. (2004a, 2005a, 2005b) and Papers I and II. Due to the small amplitudes, the phase correction is small for most stars but does slightly reduce the scatter in temperature and tighten up the sequences discussed below. None of the following discussion is affected.

Nearly all of the points representing known classical and type II Cepheids have temperatures hotter than $5400 \mathrm{~K}$ and gravities lower than $\log g=4.0$. In fact, they are clustered in an easily recognizable instability strip. Twenty-three, or about $8 \%$, of the program stars fall within this region. We shall refer to these as Cepheid strip candidates to distinguish them from known Cepheids.

In Figure 5(b), we have superimposed the MILES stars on the same plot for comparison. Several conclusions can be drawn from an examination of this plot: (1) the MILES stars are

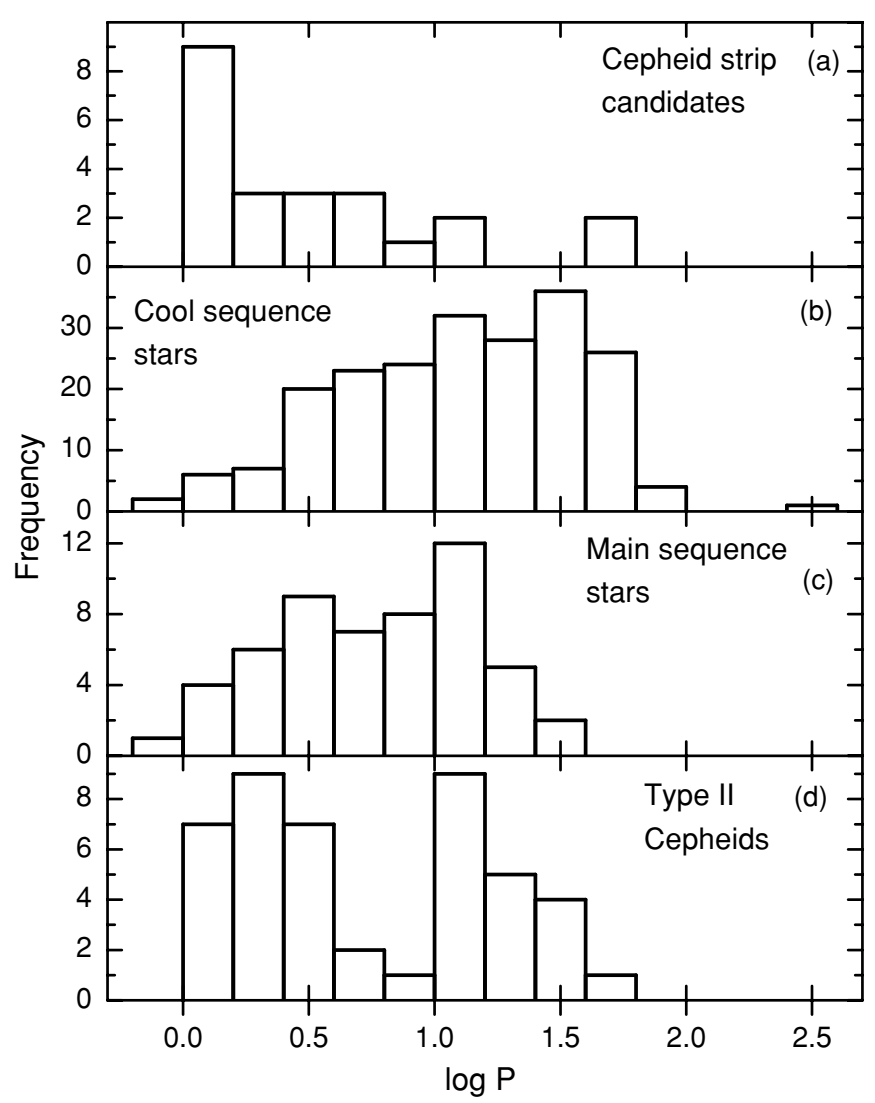

Figure 6. Frequency distributions of $\log P$ : (a) Cepheid strip candidates, (b) cool sequence stars, (c) main-sequence stars, and (d) type II Cepheids.

sufficiently well distributed in $T_{\text {eff }}$ and $\log g$ to justify their use as the reference set, (2) cooler than about $6100 \mathrm{~K}$ our sample contains approximately 57 main-sequence stars, and (3) the sequence of stars from about $(5200 \mathrm{~K}, 4.0)$ to $(3600 \mathrm{~K}, 0.6)$ is made up of stars similar to those that define the red subgiant and giant branches of the MILES sample. We shall refer to these latter two groups of stars as the main-sequence stars and the cool sequence stars, respectively. For the purposes of the discussion, we have drawn lines in Figure 5(b) to delineate the three types of stars. ${ }^{7}$

The temperature and gravity offsets of the known Cepheids relative to the standard stars discussed in Section 3.3.3 were $480 \mathrm{~K}$ and 1.18 , respectively. Shifting the Cepheid strip stars toward lower temperatures and gravities relative to the other two groups by these amounts would only diminish the separation of the sequences slightly. On the other hand, it would reduce the gravities of the stars at lower end of the Cepheid strip from around $\log g=4$ to about $\log g=3.0$. This is more reasonable for such stars and indicates that we can consider stars throughout this region to be possible Cepheids. Thus, the uncertainties concerning the temperatures and gravities of the variables relative to the standard stars found in Figure 3 will have no effect on our conclusions.

Figures 6, 7, and 8 show the distributions of the periods, amplitudes, and metallicities for each type of star. In Figures 6(d) and 7(d), we show distributions for known type II Cepheids taken from Figures 4 and 5 of Paper I. In Figures $8(\mathrm{~d})$ and (e), metallicity distributions are plotted for

\footnotetext{
7 For reference, the dividing lines are given by $\log g=0.00257 T_{\text {eff }}-11.41$
} and $\log g=-0.000157 T_{\text {eff }}+5.06$. 


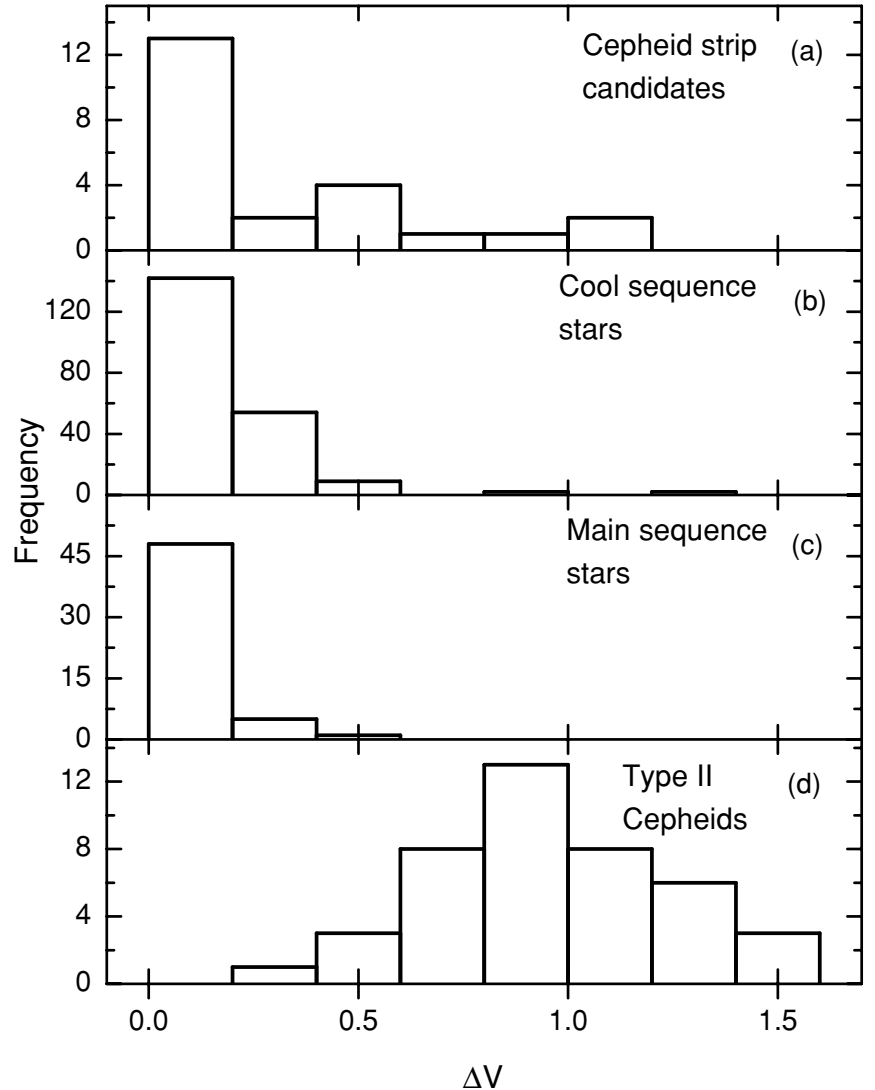

Figure 7. Distributions of amplitudes for various sets of stars: (a) Cepheid strip candidates, (b) cool sequence stars, (c) main-sequence stars, and (d) type II Cepheids.

type II Cepheids from Table 1 and for field type II Cepheids from Harris (1981b).

In Figure 9, we have plotted the same information as in Figure 5(a) with different symbols; the open circles now represent spectra with a negative $\mathrm{H} \alpha$ index and filled circles represent the remainder. The division at an index of zero does not strictly discriminate between emission line stars from those with a pure absorption line but it is a convenient place to separate stars with strong emission.

Figure 10 shows examples of light curves for each type of star. The large symbols represent Behlen Observatory data from Papers I and II (taken between 2003 September and 2008 July), while the small symbols represent data from the first release of the NSVS (Wozniak et al. 2004, taken between 1999 April and 2000 March). Although most of these curves have been shown in previous publications, they are grouped here so as to allow a comparison among the three types of stars. We will refer to these figures in the following discussion of each of the three groups.

\subsection{The Cepheid Strip Candidates}

A Kolmogorov-Smirnov test fails to demonstrate a significant difference between the period distributions of the Cepheid strip candidates (Figure 6(a)) and the known type II Cepheids (Figure 6(d)). That is, although the two histograms appear somewhat different, the number of stars is too small to demonstrate statistically whether or not the candidates have the same period distribution as the known Cepheids; it tells us nothing about the Cepheid strip candidates.

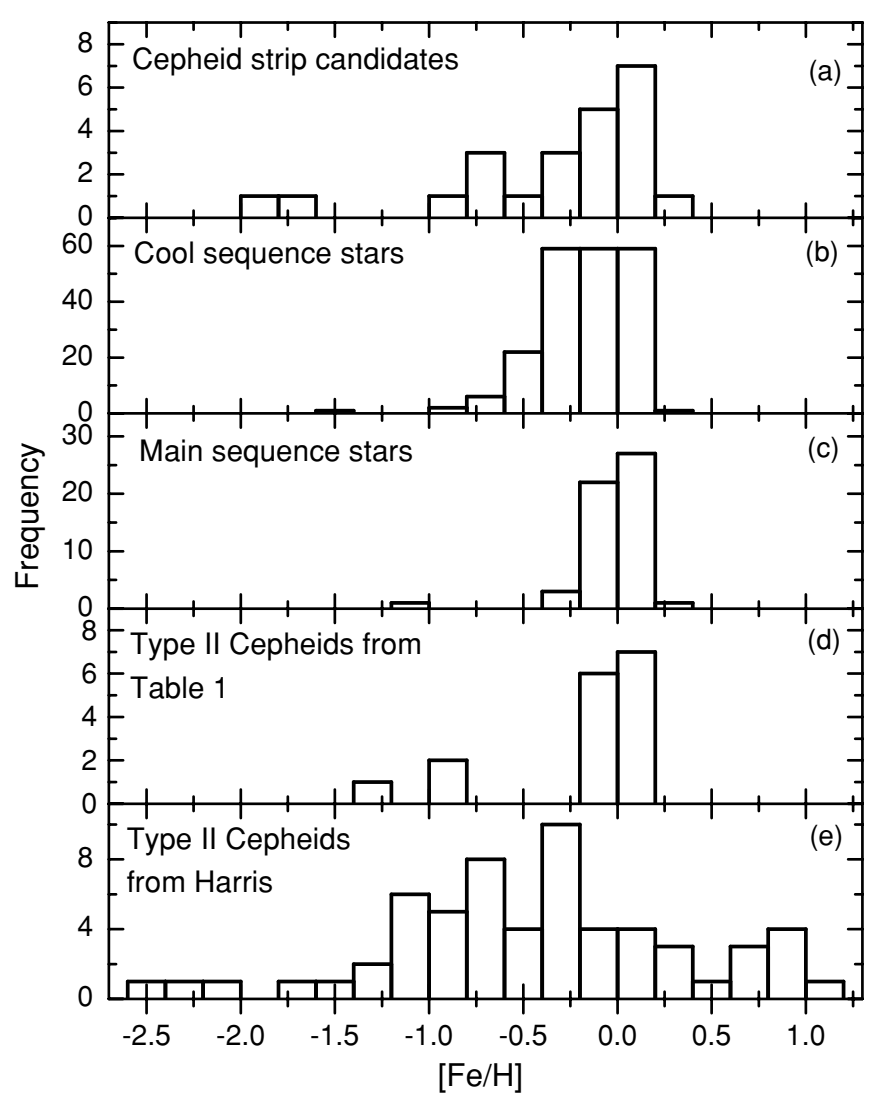

Figure 8. Distributions of metallicity for various sets of stars: (a) Cepheid strip candidates, (b) cool sequence stars, (c) main-sequence stars, (d) known type II Cepheids from Table 1, and (e) field type II Cepheids from Harris (1981b).

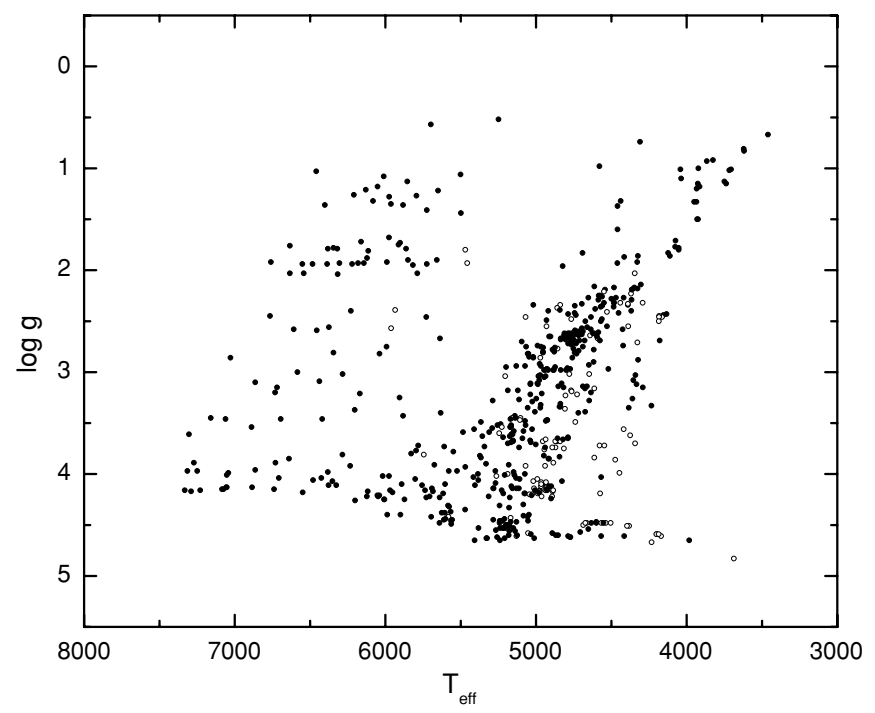

Figure 9. $\log g$ plotted against $T_{\text {eff. }}$. Open circles indicate spectra with $\mathrm{H} \alpha$ emission (negative $\mathrm{H} \alpha$ index), while closed circles indicate spectra with weak or absent $\mathrm{H} \alpha$ emission.

Figure 7(a) shows that nearly two-thirds of the Cepheid strip candidates have amplitudes less than 0.2 mag. This is in sharp contrast with the known type II Cepheids, Figure $7(\mathrm{~d})$, where only one of the 42 stars has amplitude less than 0.4 mag.

Figure 8(a) shows that the Cepheid strip candidates cluster around solar metallicity although a couple are significantly metal 

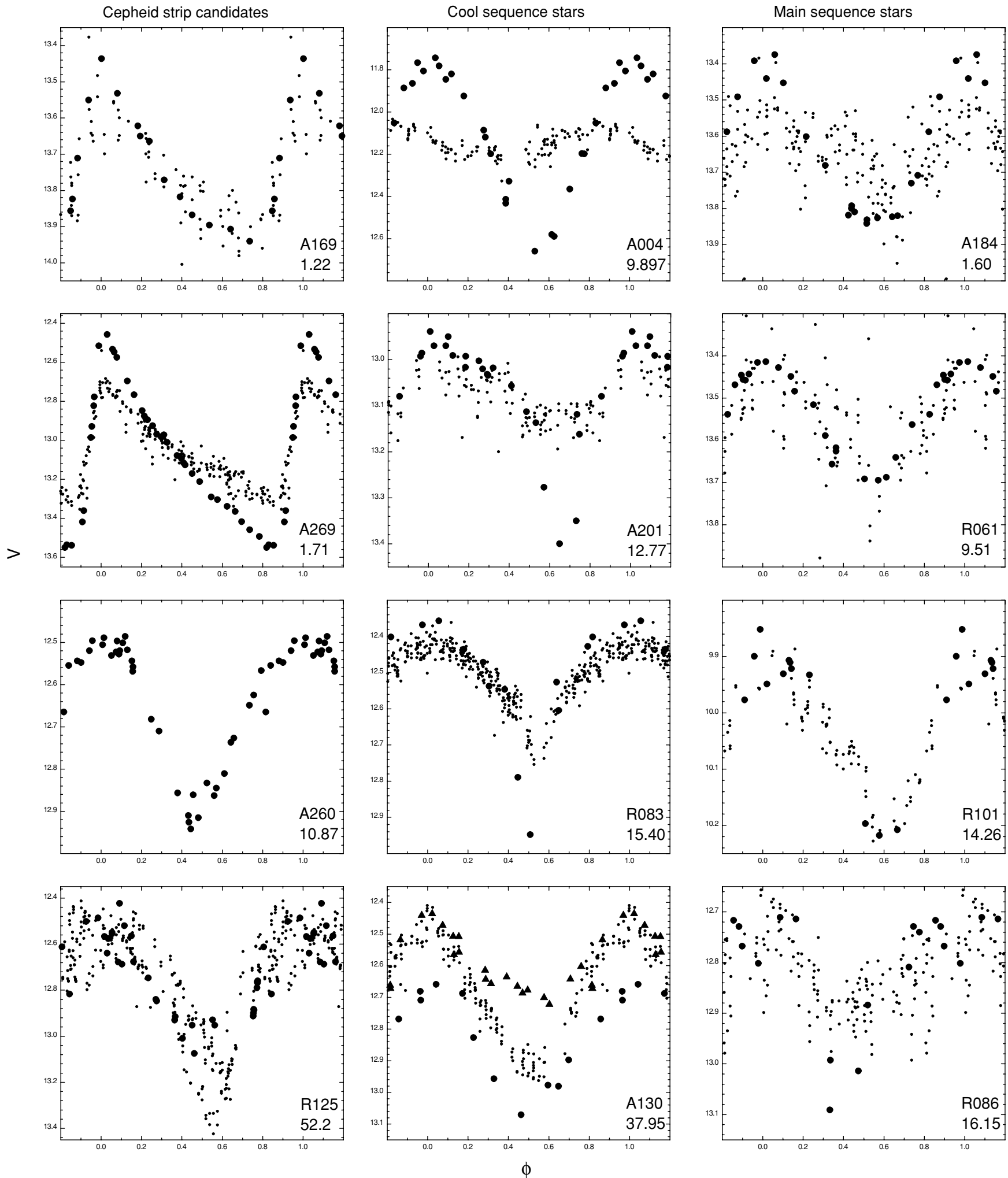

Figure 10. Examples of light curves for stars discussed in the text. The star identification and period is given to the lower right of each light curve. The left column contains Cepheid strip candidates, the central column cool sequence stars, and the right column main-sequence stars. Large symbols represent Behlen Observatory data, while small symbols represent data from the NSVS. For A130, circles represent data from the first season it was observed (2005 November to 2006 May) and triangles represent data from the second season (2006 November to 2007 May).

poor. Figures $8(\mathrm{~d})$ and (e) show a similar preponderance of solar metallicity and moderately metal-poor stars. Thus, our stars are consistent with the metallicities of known field type II Cepheids.

In Figure 9, only four spectra of stars in the Cepheid strip region exhibit hydrogen line emission. They represent two stars with two spectra each: SZ Mon and R125. The former is a type II Cepheid with a period of $16.33 \mathrm{~d}$, while the latter is a suspected large-amplitude type II Cepheid with a period of 52.2 d. Such stars are known to have hydrogen emission at some phases (Schmidt et al. 2004b). 
In Figure 10, the light curves of A169 and A269 show the rapid rise and slow descent typical of short-period type II Cepheids (for example, see Figure 1(a) of Schmidt et al. 2005a). The smaller amplitudes of the NSVS light curves compared with those from Behlen Observatory are due to the different effective wavelengths. Aside from that, the shapes of the light curves are very similar, indicating that the pulsation is stable over periods of at least a few years. The light curves of A260 and R125 differ in appearance but are quite similar to type II Cepheids of similar period such as AL Lyr, V2338 Oph, and ET Vul (Schmidt et al. 2004a). Note that the scatter in the light curve of R125 is due to cycle-to-cycle changes that are especially obvious in the NSVS data.

There is no reason that the division between RR Lyrae stars and type II Cepheids should occur at an arbitrary period of one day. Thus, we might question whether some of the shorter period stars in the Cepheid strip region might be long-period RR Lyrae stars. Andrievsky et al. (2010) pointed out that the longest period RR Lyrae are also the most metal poor; there are virtually none with periods longer than 0.8 days more metal rich than $[\mathrm{Fe} / \mathrm{H}]=-1.0$. The metallicities of the short-period Cepheid strip stars in our sample are all more metal rich than that with the exception of three stars, A169, R203 and A269. Thus, only three of the Cepheid strip stars are possibly RR Lyrae stars.

These points all support the conclusion that the Cepheid strip candidates identified in Figure 5(b) are in fact type II Cepheids.

Figure 7(a) shows that small-amplitude type II Cepheids exist in significant numbers. Previous investigators have commented on the lack of such objects (see Percy 2007, for example) and they are absent from the GCVS. This no doubt arose from a bias against the discovery of small-amplitude stars prior to the availability of the large area photometric surveys.

The 23 type II Cepheids discovered fall far short of the $\sim 560$ expected. Given that the expectation was based on globular cluster Cepheids which are almost all more metal poor than $[\mathrm{Fe} / \mathrm{H}]=-1.25$ and have large amplitudes, the discrepancy is even greater. This raises the question of the relationship between the globular cluster Cepheids and the field type II Cepheids and the origin of the field stars.

\subsection{The Cool Sequence Stars}

The periods of the cool sequence stars, Figure 6(b), cover a wide range with a histogram that increases steadily to periods around $60 \mathrm{~d}(\log P=1.8)$. This supports the conclusion above, based on the effective temperature and gravity, that these stars are not Cepheids.

The amplitudes are mostly small, Figure 7(b). However, of the 209 stars, 13 have amplitudes greater than 0.4 mag. Their appearance among the cool sequence stars is surprising in that most were identified as type II Cepheids in Papers I and II on the basis of photometry.

The metallicities of these stars, Figure 8(b), are solar or mildly metal poor with the single exception of R094 at $[\mathrm{Fe} / \mathrm{H}]=-1.56$. The atmospheric parameters from the two spectra of this star stand out from the other cool sequence stars in Figure 5 at $\left(T_{\text {eff }}, \log g\right)$ of $(4309 \mathrm{~K}, 0.74)$ and $(4579 \mathrm{~K}$, $0.98)$. Its amplitude is $\Delta V \sim 0.13$ and the pulsation rather irregular. Given these factors, this variable may resemble the postasymptotic giant branch or pre-planetary nebula stars described, for example, by Sasselov (1993) and Hrivnak et al. (2010).

In Figure 9, it is evident that hydrogen emission is common among the cool sequence variables; about $20 \%$ of the stars have an $\mathrm{H} \alpha$ index smaller than zero in at least one spectrum. About two-thirds of the stars with emission have periods less than $10 \mathrm{~d}$ which suggests that many of them, and possibly some of the long-period stars, are interacting binaries.

The light curves show a variety of forms as indicated by the examples in the second column of Figure 10. The light curve of A004 is similar to some type II Cepheids such as IX Cas (Schmidt et al. 2004a). However, the difference in amplitude between the NSVS photometry and the Behlen photometry is too great to be accounted for by the differing bandpasses; we conclude that the amplitude increased significantly over several years. We also note a shift in the phase of maximum that indicates a period change or fluctuation. The minima of A201 and R083 in the Behlen photometry are quite narrow and might be suggestive of eclipses. However, the earlier NSVS light curve for A201 shows nearly sinusoidal variation without a narrow minimum, while the minimum in R083 was much shallower than in the Behlen photometry. Thus, the history of the variations does not support the interpretation of these stars as eclipsing binaries. They merit further long-term observation. Poor repetition from cycle to cycle or over long periods is not uncommon among the cool sequence stars and is illustrated by A130.

\subsection{The Main-sequence Stars}

The generally short periods (Figure 6(c)), small amplitudes (Figure 7(c)), prevalence of $\mathrm{H} \alpha$ emission (Figure 9), and rather sinusoidal light curves (Figure 10) suggest that many of these stars are ellipsoidal, eclipsing, or rotating variables. Others may be active, late-type dwarfs. Unlike R125 in the lower left panel of Figure 10 and the stars in the second column, the main-sequence stars show little evidence of light curve changes. Further observations are needed to ascertain the nature of individual stars.

\section{CONCLUSIONS}

1. Many stars that appeared to be type II Cepheids on the basis of photometry turn out to be on the red subgiant and giant branches.

2. Many fewer type II Cepheids were discovered than expected. Unless there are serious, unknown selection effects, further examination of the origin and evolutionary history of these stars is required. As noted in Section 4.1, the field type II Cepheids have a very different metallicity distribution than those in clusters. This further emphasizes the need to reconsider their origin.

3. A significant number of low-amplitude type II Cepheids were found. This is contrary to the previous belief that such stars did not exist.

4. Variables were found all along the red subgiant and giant region of the $T_{\text {eff }}-\log g$ diagram. Many are likely binaries but light curve changes make this unlikely for others.

5. Variation of main-sequence stars in our sample appears to be mostly due to binarity or stellar activity but further investigation is warranted.

6. This work shows that the classification of variables based on photometric surveys of limited accuracy and temporal coverage is very uncertain. Automatic classification of variables from surveys is useful for identifying stars of interest. However, it is suggested that physical classes should not be assigned in this way. Rather, the light curves should be sorted into descriptive groups and terms 
suggestive of particular evolutionary histories or physical properties should await more detailed follow-up studies.

We thank the staff members of Kitt Peak National Observatory for their very able help in obtaining the data used here. D.R. and L.T.-L. were supported during this work by the Undergraduate Creative Activities and Research program at the University of Nebraska. The University of Nebraska Research Council provided travel funds. Dr. Patricia Sánchez-Blázquez provided Lick/IDS indices for the stars from the MILES survey. This was very helpful for this work and we are grateful for her help and for the efforts of the entire team that produced MILES. We acknowledge with thanks the observations of $\mathrm{W}$ Vir from the AAVSO International Database contributed by observers worldwide and used in this research. We are grateful to the referee, George Wallerstein, for directing our attention to the importance of the differences between the metallicities of the field and the cluster type II Cepheids.

Facility: KPNO:2.1m

\section{REFERENCES}

Akerlof, C., et al. 2000, AJ, 119, 1901

Andrievsky, S. M., Kovtyukh, V. V., Wallerstein, G., Korotin, S. A., \& Huang, W. 2010, PASP, 122, 877

Cenarro, A. J., et al. 2007, MNRAS, 374, 664

Diethelm, R. 1990, A\&A, 239, 186

Friel, E. D. 1987, AJ, 93, 1388

Harris, H. C. $1981 \mathrm{a}$, AJ, 86, 707

Harris, H. C. 1981 b, AJ, 86, 719

Hrivnak, B. J., Lu, W., Maupin, R. E., \& Spitzbart, B. D. 2010, ApJ, 709, 1042
Jones, J. B., Wyse, R. F. G., \& Gilmore, G. 1995, PASP, 107, 632

Kovtyukh, V. V., Soubiran, C., Luck, R. E., Turner, D. G., Belik, S. I., Andrievsky, S. M., \& Chekhonadskikh, F. A. 2008, MNRAS, 389, 1336

Kovtyukh, V. V., Wallerstein, G., \& Andrievsky, S. M. 2005, PASP, 117, 1173

Maas, T., Giridhar, S., \& Lambert, D. L. 2007, ApJ, 666, 378

Massey, P., DeVeny, J., Jennuzi, B., \& Carder, E. 2000, Low-to-Moderate Resolution Optical Spectroscopy Manual for Kitt Peak (Tucson, AZ: NOAO)

Percy, J. R. 2007, Understanding Variable Stars (Cambridge: Cambridge Univ. Press), 164

Pojmanski, G., Pilecki, B., \& Szczygiel, D. 2005, Acta Astron., 55, 275

Robinson, S. E., Strader, J., Ammons, S. M., Laughlin, G., \& Fischer, D. 2006, ApJ, 637, 1102

Sánchez-Blázquez, P., et al. 2006, MNRAS, 371, 703

Sasselov, D. D. 1993, in IAU Symp. 155, Planetary Nebulae, ed. R. Weinberger \& A. Acker (Dordrecht: Kluwer), 259

Schmidt, E. G., Hemen, B., Rogalla, D., \& Thacker-Lynn, L. 2009, AJ, 137, 4598 (Paper II)

Schmidt, E. G., Johnston, D., Langan, S., \& Lee, K. M. 2004a, AJ, 128, 1748

Schmidt, E. G., Johnston, D., Langan, S., \& Lee, K. M. 2005a, AJ, 129, 2007

Schmidt, E. G., Johnston, D., Langan, S., \& Lee, K. M. 2005b, AJ, 130, 832

Schmidt, E. G., Johnston, D., Lee, K. M., Langan, S., Newman, P. R., \& Snedden, S. A. 2004 b, AJ, 128, 2988

Schmidt, E. G., Langan, S., Rogalla, D., \& Thacker-Lynn, L. 2007, AJ, 133, 665 (Paper I)

VandenBerg, D. A., \& Clem, J. L. 2003, AJ, 126, 778

Wallerstein, G. 2002, PASP, 114, 689

Wallerstein, G., Kovtyukh, V. V., \& Andrievsky, S. M. 2008, PASP, 120 361

Worthey, G., Faber, S. M., Gonzalez, J. J., \& Burstein, D. 1994, ApJS, 94, 687

Worthey, G., \& Jowett, K. J. 2003, PASP, 115, 96

Worthey, G., \& Ottaviani, D. L. 1997, ApJS, 111, 377

Wozniak, P. R., et al. 2004, AJ, 127, 2436 